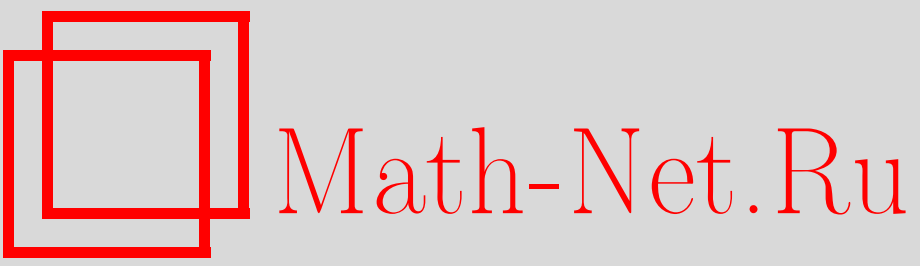

В. В. Зудилин, Одно из чисел $\zeta(5), \zeta(7), \zeta(9), \zeta(11)$ иррационально, $У M H, 2001$, том 56, выпуск 4, 149-150

DOI: https://doi.org/10.4213/rm427

Использование Общероссийского математического портала Math-Net.Ru подразумевает, что вы прочитали и согласны с пользовательским соглашением

http://www . mathnet.ru/rus/agreement

Параметры загрузки:

IP : 18.207 .199 .55

26 апреля 2023 г., 16:07:15 


\section{ОДНО ИЗ ЧИСЕЛ $\zeta(5), \zeta(7), \zeta(9), \zeta(11)$ ИРРАЦИОНАЛЬНО}

\section{В.В. Зудилин}

В настоящей заметке мы устанавливаем следующий резултат.

Теорема. По крайней мере одно из четырех чисел $\zeta(5), \zeta(7), \zeta(9)$ и $\zeta(11)$ иррационально.

Для доказательства мы используем обобщение конструкции линейных приближающих форм от значений дзета-функции Римана в нечетных точках, предложенной Т. Ривоалем в [1]. Именно с помощью этой аналитической конструкции в работе [1] была доказана бесконечность множества иррациональных чисел среди $\zeta(3), \zeta(5), \zeta(7), \ldots$; резултат об иррациональности одного из девяти чисел $\zeta(5), \zeta(7), \ldots, \zeta(21)$ был получен независимо Ривоалем [2] и автором [3]. Напомним также, что иррациональность числа $\zeta(3)$ установлена Р. Апери [4]

Зафиксируем нечетные числа $q$ и $r, q \geqslant r+4$, и набор целых положителшных параметров $\eta_{0}, \eta_{1}, \eta_{2}, \ldots, \eta_{q}$, удовлетворяющих условиям $\eta_{1} \leqslant \eta_{2} \leqslant \cdots \leqslant \eta_{q}<\eta_{0} / 2$ и

$$
\eta_{1}+\eta_{2}+\cdots+\eta_{q} \leqslant \eta_{0} \cdot \frac{q-r}{2} .
$$

Для каждого целого $n>0$ определим целочисленньй набор

$$
h_{0}=\eta_{0} n+2, \quad h_{j}=\eta_{j} n+1, \quad j=1, \ldots, q,
$$

и рассмотрим рациональную функцию

$$
\begin{aligned}
R_{n}(t):= & \left(h_{0}+2 t\right) \cdot \prod_{j=1}^{r} \frac{1}{\left(h_{j}-1\right) !} \frac{\Gamma\left(h_{j}+t\right)}{\Gamma(1+t)} \cdot \prod_{j=1}^{r} \frac{1}{\left(h_{j}-1\right) !} \frac{\Gamma\left(h_{0}+t\right)}{\Gamma\left(1+h_{0}-h_{j}+t\right)} \\
& \times \prod_{j=r+1}^{q}\left(h_{0}-2 h_{j}\right) ! \frac{\Gamma\left(h_{j}+t\right)}{\Gamma\left(1+h_{0}-h_{j}+t\right)},
\end{aligned}
$$

а также соответствующую ей величину

$$
F_{n}:=\frac{1}{(r-1) !} \sum_{t=0}^{\infty} R_{n}^{(r-1)}(t)
$$

(согласно (1) вьполнено $R_{n}(t)=O\left(t^{-2}\right)$, что обеспечивает сходимость ряда в правой части $\left.(2)\right)$.

Положим $m_{j}=\max \left\{\eta_{r}, \eta_{0}-2 \eta_{r+1}, \eta_{0}-\eta_{1}-\eta_{r+j}\right\}$ для $j=1, \ldots, q-r$ и определим целую величину

$$
\Phi_{n}:=\prod_{\sqrt{\eta_{0} n}<p \leqslant m_{q-r} n} p^{\varphi(n / p)},
$$

где в произведении участвуют толшко простые числа и

$$
\begin{aligned}
\varphi(x):=\min _{0 \leqslant y<1}\left(\sum_{j=1}^{r}\left(\lfloor y\rfloor+\left\lfloor\eta_{0} x-y\right\rfloor-\left\lfloor y-\eta_{j} x\right\rfloor-\left\lfloor\left(\eta_{0}-\eta_{j}\right) x-y\right\rfloor-2\left\lfloor\eta_{j} x\right\rfloor\right)\right. \\
\left.+\sum_{j=r+1}^{q}\left(\left\lfloor\left(\eta_{0}-2 \eta_{j}\right) x\right\rfloor-\left\lfloor y-\eta_{j} x\right\rfloor-\left\lfloor\left(\eta_{0}-\eta_{j}\right) x-y\right\rfloor\right)\right)
\end{aligned}
$$

- целозначная неотрицательная периодическая (с периодом 1 ) функция. Через $D_{N}$ обозначим наименьшее общее кратное чисел $1,2, \ldots, N$.

Работа выполнена при частичной поддержке фонда INTAS и Российского фонда фундаментальных исследований (грант № IR-97-1904). 
Лемма 1. Величина (2) является линейной формой от $1, \zeta(r+2), \zeta(r+4), \ldots, \zeta(q-2)$ с рациональными коэффициентами; более того, справедливо включение

$$
D_{m_{1} n}^{r} D_{m_{2} n} \cdots D_{m_{q-r} n} \cdot \Phi_{n}^{-1} \cdot F_{n} \in \mathbb{Z}+\mathbb{Z} \zeta(r+2)+\mathbb{Z} \zeta(r+4)+\cdots+\mathbb{Z} \zeta(q-2) .
$$

Асимптотика величины $\Phi_{n}$ при $n \rightarrow \infty$ вьчисляется с помощью арифметического метода Чудновского-Рухадзе-Хаты (см. вычитаемое в определении постоянной $C_{1}$ из формулировки леммы 3 далее). Кроме того, согласно закону распределения простых чисел

$$
\lim _{n \rightarrow \infty} \frac{\log D_{m_{j}} n}{n}=m_{j}, \quad j=1, \ldots, q-r .
$$

Введем вспомогательную функцию

$$
\begin{aligned}
f_{0}(\tau)= & r \eta_{0} \log \left(\eta_{0}-\tau\right)+\sum_{j=1}^{q}\left(\eta_{j} \log \left(\tau-\eta_{j}\right)-\left(\eta_{0}-\eta_{j}\right) \log \left(\tau-\eta_{0}+\eta_{j}\right)\right) \\
& -2 \sum_{j=1}^{r} \eta_{j} \log \eta_{j}+\sum_{j=r+1}^{q}\left(\eta_{0}-2 \eta_{j}\right) \log \left(\eta_{0}-2 \eta_{j}\right),
\end{aligned}
$$

определенную в $\tau$-плоскости с разрезами $\left(-\infty, \eta_{0}-\eta_{1}\right]$ и $\left[\eta_{0},+\infty\right)$. Следующее утверждение, характеризующее рост линейных форм $F_{n}$ в случае $r=3$, доказывается с помощью представления величины (2) в виде комплексного интеграла по прямой Re $t=$ const с последующим применением к нему асимптотики гамма-функции и метода перевала.

ЛЕмма 2. Пусть $r=3$ и $\tau_{0}-$ нуль многочлена

$$
\left(\tau-\eta_{0}\right)^{r}\left(\tau-\eta_{1}\right) \cdots\left(\tau-\eta_{q}\right)-\tau^{r}\left(\tau-\eta_{0}+\eta_{1}\right) \cdots\left(\tau-\eta_{0}+\eta_{q}\right)
$$

$c \operatorname{Im} \tau_{0}>0$ и максимально возможным значением $\operatorname{Re} \tau_{0}$. Предположим, что выполнено $\operatorname{Re} \tau_{0}<\eta_{0} u \operatorname{Im} f_{0}\left(\tau_{0}\right) \notin \pi \mathbb{Z}$. Тогда

$$
\varlimsup_{n \rightarrow \infty} \frac{\log \left|F_{n}\right|}{n}=\operatorname{Re} f_{0}\left(\tau_{0}\right) .
$$

Если последовательность линейных форм в левой части (3) с ростом $n$ принимает ненулевые сколь угодно малше значения, то в случае $r=3$ среди чисел

$$
\zeta(5), \zeta(7), \ldots, \zeta(q-4), \zeta(q-2)
$$

имеется иррациональное. Поэтому справедливо следующее утверждение.

Лемма 3. Пусть $r=3$ и в приведенных выше обозначениях $C_{0}=-\operatorname{Re} f_{0}\left(\tau_{0}\right)$,

$$
C_{1}=r m_{1}+m_{2}+\cdots+m_{q-r}-\left(\int_{0}^{1} \varphi(x) \mathrm{d} \psi(x)-\int_{0}^{1 / m_{q-r}} \varphi(x) \frac{\mathrm{d} x}{x^{2}}\right),
$$

где $\psi(x)$ - логарифмическая производная гамма-функции. Тогда в случае $C_{0}>C_{1}$ по крайней мере одно из чисел (4) иррачионально.

Для доказательства теоремы положим $r=3, q=13$,

$$
\eta_{0}=91, \quad \eta_{1}=\eta_{2}=\eta_{3}=27, \quad \eta_{4}=29, \eta_{5}=30, \eta_{6}=31, \ldots, \eta_{12}=37, \eta_{13}=38 .
$$

Тогда $C_{0}=227.58019641 \ldots, C_{1}=226.24944266 \ldots$, и согласно лемме 3 среди чисел $\zeta(5), \zeta(7)$, $\zeta(9), \zeta(11)$ имеется иррациональное.

\section{СПИСОК ЛИТЕРАТУРЫ}

[1] T. Rivoal // C. R. Acad. Sci. Paris. Sér. I Math. 2000. V. 331. № 4. P. 267-270. [2] T. Rivoal. Propriétés diophantinnes des valeurs de la fonction zêta de Riemann aux entiers impairs. Thèse de Doctorat. Caen: Univ. de Caen, 2001. [3] В. В. Зудилин // УМН. 2001. Т. 56. № 2. C. 215-216. [4] R. Apéry // Astérisque. 1979. V. 61. P. 11-13. 